\title{
SARS-CoV-2-related encephalitis with prominent parkinsonism: clinical and FDG-PET correlates in two patients
}

\author{
Mauro Morassi ${ }^{1}\left(\mathbb{D} \cdot\right.$ Francesco Palmerini $^{2} \cdot$ Stefania Nici $^{3} \cdot$ Eugenio Magni $^{2} \cdot$ Giordano Savelli $^{4} \cdot$ Ugo Paolo Guerra $^{4}$. \\ Matteo Chieregato ${ }^{3} \cdot$ Silvia Morbelli ${ }^{5} \cdot$ Alberto Vogrig $^{6}$
}

Received: 21 December 2020 / Revised: 23 March 2021 / Accepted: 12 April 2021 / Published online: 21 April 2021

○) Springer-Verlag GmbH Germany, part of Springer Nature 2021

\begin{abstract}
Considering the similarities with other pandemics due to respiratory virus infections and subsequent development of neurological disorders (e.g. encephalitis lethargica after the 1918 influenza), there is growing concern about a possible new wave of neurological complications following the worldwide spread of SARS-CoV-2. However, data on COVID-19-related encephalitis and movement disorders are still limited. Herein, we describe the clinical and neuroimaging (FDG-PET/CT, MRI and DaT-SPECT) findings of two patients with COVID-19-related encephalopathy who developed prominent parkinsonism. None of the patients had previous history of parkinsonian signs/symptoms, and none had prodromal features of Parkinson's disease (hyposmia or RBD). Both developed a rapidly progressive form of atypical parkinsonism along with distinctive features suggestive of encephalitis. A possible immune-mediated etiology was suggested in Patient 2 by the presence of CSF-restricted oligoclonal bands, but none of the patients responded favorably to immunotherapy. Interestingly, FDG-PET/ CT findings were similar in both cases and reminiscent of those observed in post-encephalitic parkinsonism, with cortical hypo-metabolism associated with hyper-metabolism in the brainstem, mesial temporal lobes, and basal ganglia. Patient's FDG-PET/CT findings were validated by performing a Statistical Parametric Mapping analysis and comparing the results with a cohort of healthy controls $(n=48)$. Cerebrum cortical thickness map was obtained in Patient 1 from MRI examinations to evaluate the structural correlates of the metabolic alterations detected with FDG-PET/CT. Hypermetabolic areas correlated with brain regions showing increased cortical thickness, suggesting their involvement during the inflammatory process. Overall, these observations suggest that SARS-CoV-2 infection may trigger an encephalitis with prominent parkinsonism and distinctive brain metabolic alterations.
\end{abstract}

Keywords COVID-19 $\cdot$ FDG-PET/CT $\cdot$ Neuroimaging $\cdot$ Parkinson's disease $\cdot$ Parkinsonism $\cdot$ Neurological complications

Mauro Morassi

mauro.morassi@poliambulanza.it

1 Department of Diagnostic Imaging, Unit of Neuroradiology, Fondazione Poliambulanza Hospital, Via L. Bissolati 57, 25124 Brescia, Italy

2 Unit of Neurology, Fondazione Poliambulanza Hospital, Brescia, Italy

3 Unit of Medical Physics, Fondazione Poliambulanza Hospital, Brescia, Italy

4 Unit of Nuclear Medicine, Fondazione Poliambulanza Hospital, Brescia, Italy

5 Department of Health Sciences, Unit of Nuclear Medicine, Ospedale Policlinico San Martino, Genoa, Italy

6 Clinical Neurology Unit, Santa Maria della Misericordia University Hospital, Piazzale Santa Maria della Misericordia, Udine, Italy

\section{Introduction}

While SARS-CoV-2 is known to cause interstitial pneumonia and acute respiratory distress syndrome (ARDS), there is increasing evidence of diverse neurological manifestations, including encephalopathy [1,2], limbic and brainstem encephalitis [3, 4], stroke [5-7], and Guillain-Barré syndrome $[8,9]$. These presentations may reflect either direct viral infection or, more likely, inflammatory processes with dysregulation of the immune response. In addition, there is growing concern about a potential new wave of parkinsonism following SARS-CoV-2 infection, similarly to the development of encephalitis lethargica after the 1918 influenza epidemic, but very limited data exist to prove this hypothesis. In particular, the fact that only four cases of parkinsonism have been described so far [10] when more than 110 
million people contracted SARS-CoV-2 in the world renders the possibility of a similar epidemic of neurodegenerative disorders unlikely. Conversely, the occurrence of parkinsonism following a viral infection is a well-known phenomenon, in which both causal and coincidental links are possible (i.e. unmasking of underlying, pre-symptomatic, neurologic disorder) $[10,11]$.

Herein, we describe the clinical and neuroimaging findings in two patients with prominent parkinsonism due to SARS-CoV-2-related encephalitis, focusing on 2-deoxy-2$\left[{ }^{18} \mathrm{~F}\right]$ fluoro-D-glucose positron emission tomography/computed tomography (FDG-PET/CT) metabolic alterations.

\section{Materials and methods}

Two cases of COVID-19-related encephalitis diagnosed at the Fondazione Poliambulanza Hospital, Brescia, Italy from 27th February to May 15th were reviewed. The diagnosis of encephalitis followed the criteria proposed in the literature [12], while SARS-CoV-2 positivity was confirmed by a positive reverse-transcriptase polymerase-chain-reaction (RT-PCR) assay obtained from nasopharyngeal swab. The patients underwent the following clinical and neuroimaging work-up: cerebrospinal fluid (CSF) analysis including RT-PCR assay for neurotropic viruses [herpes simplex virus (HSV)-1, HSV-2, HSV-6, Epstein-Barr virus, varicellazoster virus, cytomegalovirus and enterovirus] and SARSCoV-2 (in Patient 1), onconeuronal antibody panel (antiHuD, Ri, Yo, Amphiphysin, Ma2, CV2, Recoverin, SOX1, Titin, Zic4, GAD65, Tr) and neuronal surface antigens antibodies (anti-NMDAR, AMPAR, CASPR2, LGI1, GABABR) in blood and CSF, brain magnetic resonance imaging (MRI), electroencephalogram (EEG), and FDG-PET/CT.

FDG-PET/CT images were analyzed by Statistical Parametric Mapping (SPM). The patients (parkinsonian group, $n=2$ ) were compared to a retrospectively collected, anonymized, group of healthy subjects (control group, $n=48$ ) and the results analyzed by means of a two-sample $t$ test using the SPM12 software. The SPM analysis was optimized according to the steps previously described for other neurological diseases [13]. Family-wise error rate (FWE)corrected $p<0.05$ was considered significant.

Structural MRI examinations of Patient 1 at baseline and after 79 days were acquired with $1.5 \mathrm{~T}$ GE scanner using a gradient echo $3 \mathrm{D}$ protocol according to the following parameters: $\mathrm{TR}=8.4 \mathrm{~ms}, \mathrm{TE}=3.2 \mathrm{~ms}$, flip angle $=12^{\circ}$, acquisition matrix $256 \times 256$, voxel size $1 \mathrm{~mm}^{3}$. Cortical thickness maps were obtained with FreeSurfer version 6.0 (https://surfer. nmr.mgh.harvard.edu/). Cortical thickness was calculated as the closest distance from the gray/white boundary to the gray/CSF boundary at each vertex on the tessellated surface [14]. Reconstructions were visually inspected and corrected manually. After cross-sectional processing, images were processed with the longitudinal two stage stream [15]. The percent change of thickness with respect to the thickness at the first time point was smoothed with a Gaussian kernel of $25 \mathrm{~mm}$ and is shown on the pial average surface between the two time points.

I-123 ioflupane dopamine transporter single-photon emission CT (DaT-SPECT) in one patient (Patient 1) was analyzed with Basal Ganglia matching tools V2 [16].

\section{Results}

Clinical and laboratory features of the two patients are summarized in Table 1, while complete clinical vignettes are described below.

\section{Presentation of cases}

Patient 1 was a 70 -year-old woman who presented to the Emergency Department with fever and cough for 2 days. Her past medical history included arterial hypertension and mixed anxiety-depressive disorder treated with trazodone, fluoxetine and benzodiazepines. A geriatric evaluation performed 45 days prior to admission found no evidence of neurological disorders on clinical examination and neuropsychological tests yielded normal results (MMSE: 28/30, clock drawing test correctly performed). Her body temperature at admission was $37.2^{\circ} \mathrm{C}$ with no evidence of respiratory distress $\left(\mathrm{SpO}_{2} 97 \%, \mathrm{PaO}_{2} / \mathrm{FiO}_{2} 321\right)$. Nevertheless, a chest $\mathrm{CT}$ showed multiple bilateral ground-glass opacities suggestive of SARS-CoV-2 infection. Nasopharyngeal swab was positive for SARS-CoV-2 on RT-PCR assay. Due to the moderate disease (according to WHO) with no sign of acute respiratory distress, the patient was discharged with domiciliary therapy (darunavir, ritonavir and hydroxychloroquine). During the following 2 weeks, her medical conditions worsened and she was admitted to an outside Hospital with respiratory insufficiency $\left(\mathrm{SpO}_{2} 67 \%\right)$. Repeated chest $\mathrm{CT}$ showed multiple consolidations suggestive for late stage of the disease. Following two consecutive nasopharyngeal swabs negative for SARS-CoV-2 infection, the patient was transferred to our Hospital. At admission (day 0), her body temperature was $38.3{ }^{\circ} \mathrm{C}$ and oxygen saturation $\left(\mathrm{SpO}_{2}\right)$ was $98 \%$ while receiving oxygen therapy $(12 \mathrm{~L} / \mathrm{min}$ with Venturi mask $50 \%$ ). Initial neurological examination found the patient to be alert and oriented, speech was fluent with good comprehension, and no motor weakness was present. The patient progressively developed psychomotor agitation, aggressive behavior and confusion in association with spontaneous myoclonus in the upper and lower limbs. Sleep-wake cycle inversion was also noted. Temporary treatment with haloperidol was required for controlling agitation, which gradually 
Table 1 Clinical characteristics and initial laboratory features of the patients

\begin{tabular}{lll}
\hline Clinical characteristics & Patient 1 & Patient 2 \\
\hline Age & 70 & 73 \\
Sex & F & F \\
Comorbidities & Hypertension, & Diabetes mellitus II, \\
& depression & hypertension, \\
& & depression \\
COVID-19 symptoms & Fever, & Fever \\
& cough, & \\
Chest CT & dysgeusia & Unilateral pneumonia \\
Supplemental oxygen during hospitalization & Bilateral pneumonia & Yes \\
Laboratory features & Yes & \\
Haemoglobin $(\mathrm{g} / \mathrm{dL})(12.00-16.00)$ & & 10.7 \\
Platelet count $\left(10^{9} / \mathrm{L}\right)(130-450)$ & 10.4 & 179 \\
Lymphocyte count $\left(10^{9} / \mathrm{L}\right)(0.90-5.00)$ & 749 & 0.72 \\
Neutrophil count $\left(10^{9} / \mathrm{L}\right)(1.90-7.00)$ & 1.38 & 8.37 \\
C-reactive protein $(\mathrm{mg} / \mathrm{L})(<5)$ & 5.58 & 7.8 \\
D-dimer $($ ng/mL $)(<232)$ & 195.2 & - \\
Ferritin $(\mu \mathrm{gg} / \mathrm{L})(13-150)$ & 848 & - \\
Lactate dehydrogenase $(\mathrm{U} / \mathrm{L})(125-220)$ & 888 & 279 \\
Prothrombin time activity \% $(70-120)$ & 247 & 88 \\
Creatinine $(\mathrm{mg} / \mathrm{dL})(0.55-1.02)$ & 50 & 1.55 \\
CSF cellularity $\left(\mathrm{cell} / \mathrm{mm}{ }^{3}\right)(<4)$ & 1.12 & 4 \\
CSF protein levels $(\mathrm{mg} / \mathrm{dL})(20-45)$ & 4 & 58 \\
CSF oligoclonal bands & 34 & Present \\
\hline
\end{tabular}

resolved. Conversely, myoclonus persisted and the patient also manifested a transient episode of loss of consciousness with diffuse myoclonic jerks. EEG showed bilateral thetadelta slowing with bitemporal epileptiform discharges. After this episode, treatment with levetiracetam (750 mg bid) was started with improvement of myoclonus. On day 10, brain MRI (MRI 1) did not reveal any acute alteration or contrastenhanced areas within the brain and/or meninges. CSF was negative for both neurotropic viruses and SARS-CoV-2 on RT-PCR assay. Two nasopharyngeal swabs (day 11 and day 14) confirmed SARS-CoV-2 infection negativity. Brain FDG-PET/CT, performed on day 20, showed diffuse cortical hypo-metabolism (with relative sparing of sensorimotor areas) (Fig. 1a). Whole-brain voxel-based SPM analysis revealed that hypo-metabolism reached highest significance in the precuneus (Brodmann area (BA) 7 and 19) and right posterior parietal cortex (BA 40) while hyper-metabolism was noted within the bilateral putamen and medial temporal lobe, cingulate gyrus, brainstem and within the vermianparavermian areas of the cerebellum. On the same day, the patient was discharged with a domiciliary therapy consisting of antiepileptic drugs.

On day 31 after hospital discharge, she developed a generalized tonic-clonic seizure and was readmitted. Neurological evaluation showed new-onset, generalized (particularly axial) hypertonia, cogwheel rigidity and loss of spontaneous movements of right limbs, bradykinesia at finger tapping, hypomimia, hypophonia and ophthalmoparesis. On further questioning, no previous history of hyposmia (neither prior to COVID-19, nor after the infection) was recalled, and no elements compatible with previous REM behavior disorder (RBD) emerged. Chest XR showed no lung parenchymal lesions. CSF examination demonstrated decreased amyloid $\beta 42$ (225 ng/L, pathologic values $<530 \mathrm{ng} / \mathrm{L}$ ) and increased total Tau protein (454 ng/L, pathologic values $>350 \mathrm{ng} / \mathrm{L})$. Onconeuronal antibody panel and neuronal surface antigens antibodies were all negative. Antiepileptic drugs (levetiracetam $750 \mathrm{mg}$ bid, valproate $500 \mathrm{mg}$ tid), clonazepam (0.5 mg q.d.), carbidopa/levodopa (100/25 mg q.i.d.), corticosteroids and intravenous immunoglobulins (IVIGs) $0.4 \mathrm{~g} / \mathrm{Kg} / \mathrm{die}$ (from day 13 to day 17 ) were administered. After controlling seizures, the patient was discharged. During the following 11 weeks, focal, fixed dystonia of the right upper limb was also noted, while a modest effect of levodopa was noticed on the other parkinsonian features. Myoclonus was no more detectable after dual antiepileptic therapy, but the patient showed a rapid deterioration of her cognitive functions (12/30 MMSE). Brain MRI (MRI 2 performed 79 days from baseline examination) showed slight enlargement of the ventricular system as compared to the previous examination. Cerebral cortical thickness analysis showed negative variations, suggestive for cortical thinning, 
Fig. 1 Brain FDG-PET findings in the patients and comparison with a control group. a-b Brain FDG-PET data of Patient 1 (a) and Patient 2 (b) displayed in spectrum color scale. The images show diffuse cortical hypo-metabolism with relatively spared metabolism in the sensorimotor cortex. c-d Voxel-based SPM analysis, comparison between the two patients and 48 healthy controls (FWE-corrected $p<0.05$ ); regions of significant difference were color-graded in terms of $Z$ values. The analyses showed hypo-metabolism within bilateral dorso-lateral prefrontal cortex, right caudate nucleus and bilateral posterior parietal cortex (c). Relative hypermetabolism was evident in the mesiotemporal cortex, basal ganglia, brainstem and cerebellum on both hemispheres (d)
FDG-PET

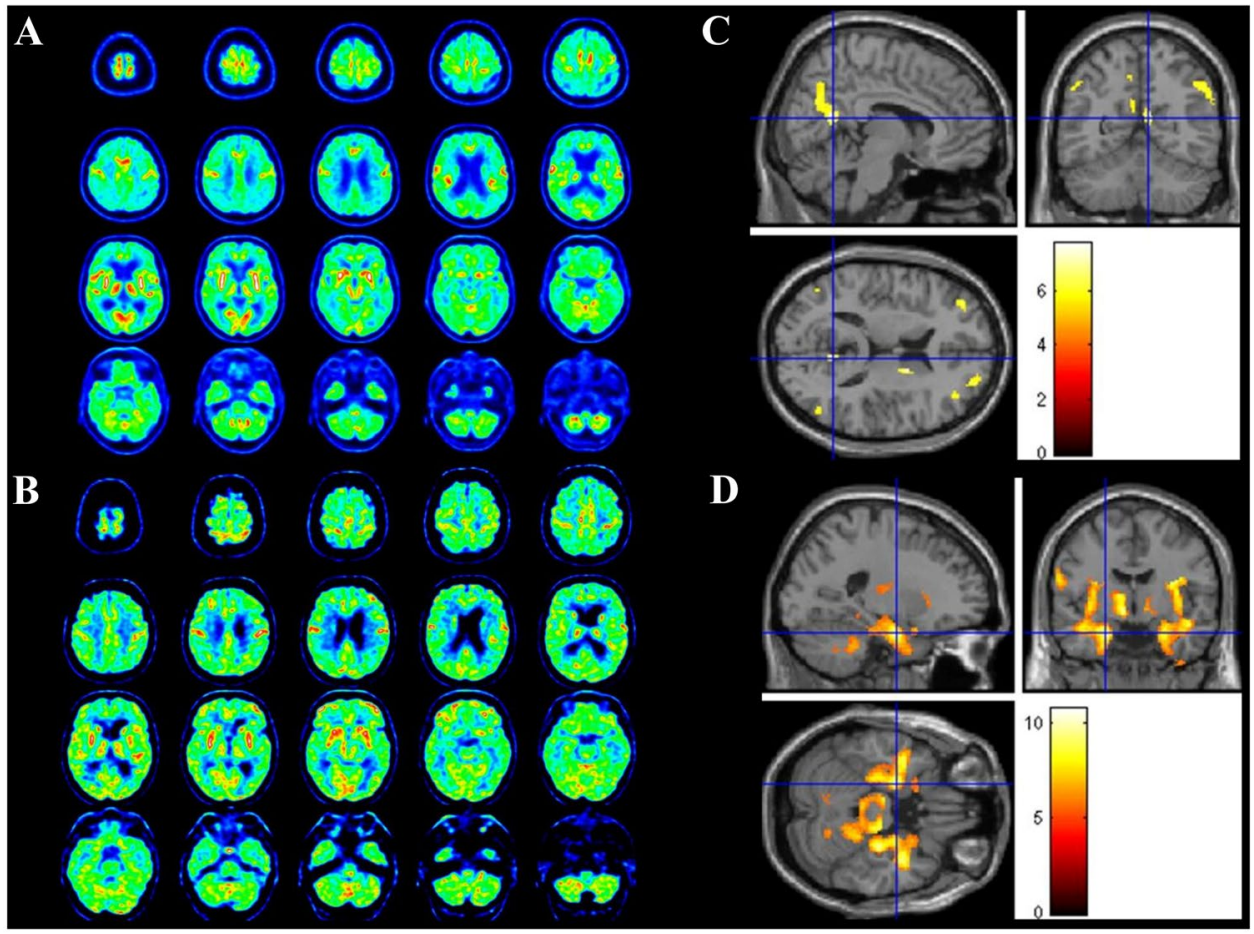

within bilateral mesial aspect of frontal superior gyrus, bilateral post-central gyrus, precuneus on the right side, focal areas within the bilateral occipital lobe, and olfactory/rectal gyrus on the right side. Positive variations, due to relatively increased cortical thickness, were noted within bilateral inferior frontal gyrus, insula, temporal pole, bilateral superior temporal gyrus, parahippocampal gyrus and anterior cingulate cortex (Fig. 2). [123I]-ioflupane DaT-SPECT was performed and confirmed a bilateral decrease in presynaptic dopamine uptake asymmetrically involving both putamina, more severe on the left side (Fig. 3). On last follow-up performed 9 months after presentation, the patient was able to walk only with aid [Modified Rankin Scale $(\mathrm{mRS})=4$ ] and severely affected in activities of daily living $(\mathrm{ADL}=3 / 6)$. Late-day confusion (sundowning) was also reported by the patient's daughter.

Patient 2 was a 73-year-old woman who presented with a post-traumatic sub-galeal left frontal hematoma. Her past medical history included type II diabetes, arterial hypertension and mixed anxiety-depressive disorder with psychotic symptoms treated with olanzapine and amilsulpride. No previous history of neurological disorders, hyposmia, or symptoms compatible with RBD was reported. The patient initially did not complain cough or dyspnea but had fever $\left(38.8{ }^{\circ} \mathrm{C}\right)$ and tested positive for RT-PCR assay from nasopharyngeal swab. Chest CT showed minimal ground-glass opacities within the right lung. During the previous 20 days of diffuse tremors, impaired posture and balance with repeated falls were reported by the daughter. At admission,

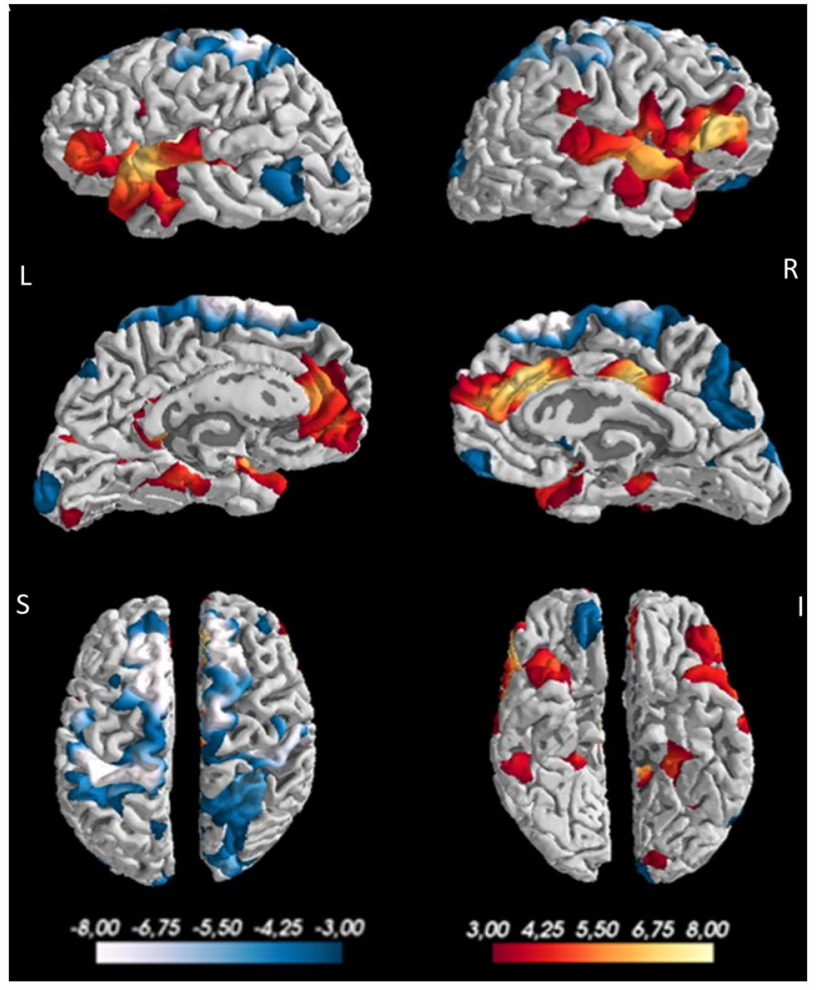

Fig. 2 Brain cortical thickness map. Brain cortical thickness map representing percentage variations of cortical thickness between the two time points (MRI 1 and MRI 2). Blue to white: areas with relative decrease of cortical thickness; red to yellow: areas with relative increase of cortical thickness. L: left hemisphere; R: right hemisphere; S: superior view; I: inferior view 

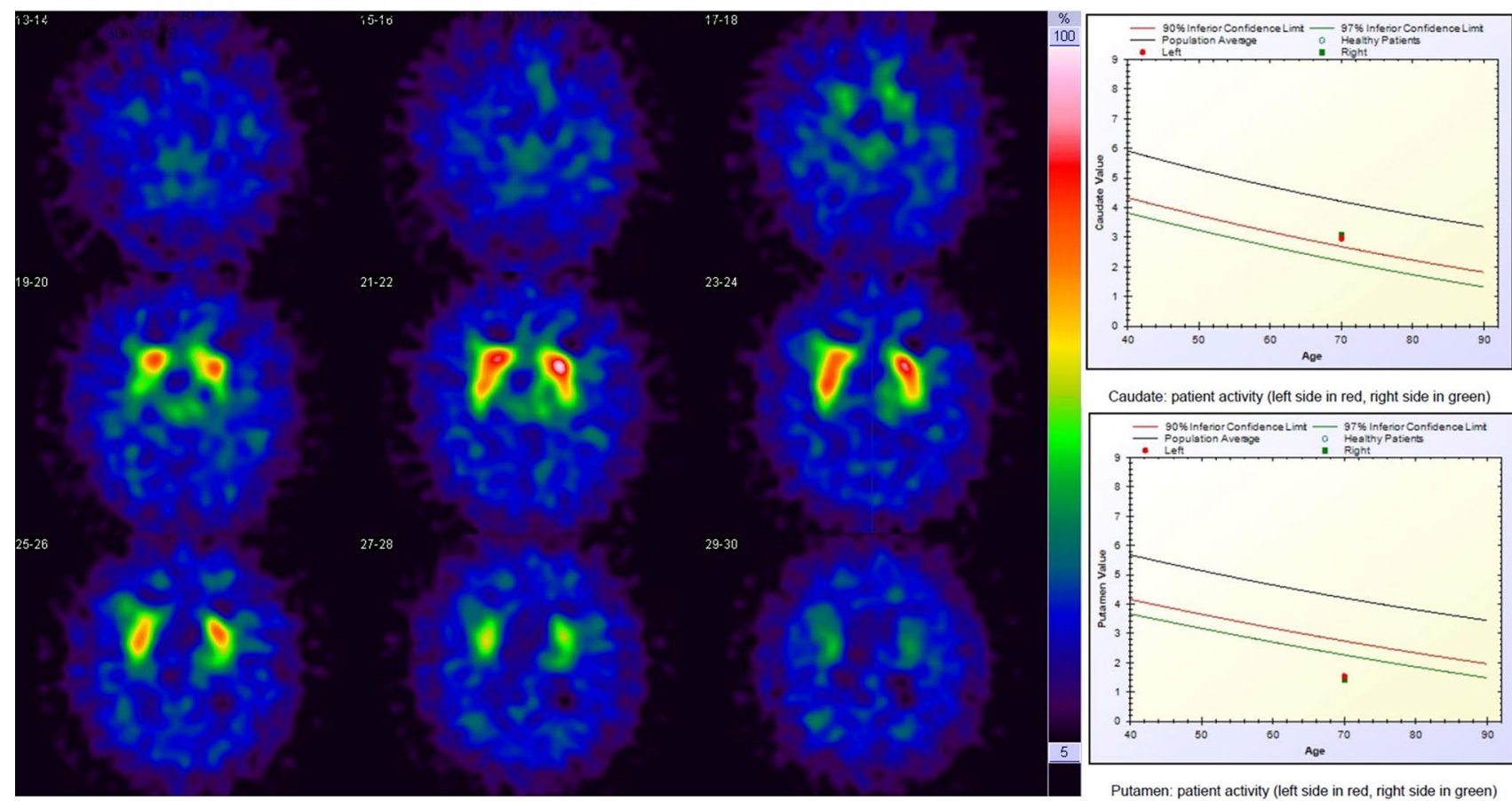

Caudate: patient activity (left side in red, right side in green)

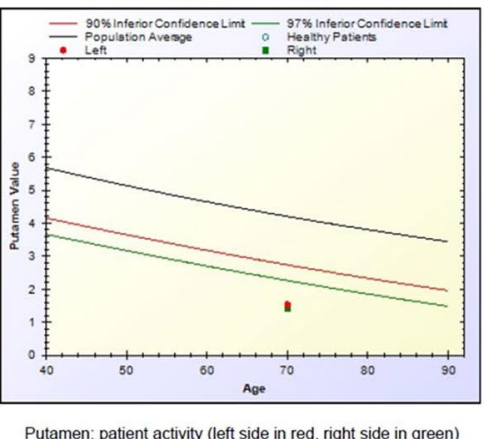

Fig. 3 DaTscan SPECT. [123I]-ioflupane (DaTscan) SPECT showed a bilaterally reduced nigrostriatal absorption affecting the putamina, more severe on the left side. Basal ganglia alterations were confirmed by semiquantitative analysis with Basal Ganglia matching tools V2

the patient was alert and comprehended simple commands, but severe anarthria was noticed. She had a slight limitation of vertical gaze. The rest of the neurological examination revealed a bilateral hypokinetic-rigid syndrome. Loss of spontaneous movements and moderate cogwheel rigidity enhanced by the Froment's maneuver were observed. Frank hypomimia with diminished blinking as well as glabellar tap sign were also documented. The following days the patient was lethargic with no or limited responsiveness to external stimuli. Neuroleptics were discontinued without improvement of the clinical syndrome. EEG showed generalized theta slowing with sharp waves over the right fronto-temporal region. Brain MRI did not reveal any acute alteration or contrast-enhanced areas within the brain and/or meninges. CSF investigations showed increased protein content (Table 1) and four oligoclonal bands. Onconeuronal antibody panel and neuronal surface antigens antibodies were negative. Brain FDG-PET/CT (day 14) showed diffuse cortical hypo-metabolism with relative sparing of sensorimotor areas (Fig. 1b). Whole-brain voxel-based SPM analysis revealed that hypo-metabolism reached highest significance in the right posterior parietal cortex (BA $40 \mathrm{~A}$ ), while relative hyper-metabolism was noted within bilateral medial temporal lobe and the brainstem.

The patient was treated with amantadine (100 mg bid,) and levodopa/carbidopa (up to 100/25 mg qid). Corticosteroids were introduced twice (from day 3 to day 6 and from day 10 to day 13) as well as IVIGs $0.4 \mathrm{~g} / \mathrm{Kg} / \mathrm{die}$ (from day
13 to day 17). The clinical conditions did not significantly improve and 30 days after discharge, the patient died of medical complications (infected bedsores and aspiration pneumonia).

\section{Comparison between FDG-PET/CT findings in the patients and a control group}

The analyses showed hypo-metabolism in a widespread cerebral network including the dorso-lateral prefrontal cortex, anterior cingulate, right caudate nucleus and bilateral posterior parietal cortex in the patients as compared to healthy controls (Fig. 1c). Relative hyper-metabolism was evident in the mesiotemporal cortex, basal ganglia, brainstem and cerebellum on both hemispheres (Fig. 1d).

\section{Discussion}

We report the clinical evolution and FDG-PET/CT findings in two patients who developed encephalitis and prominent movement disorders following SARS-CoV-2 infection. The association of neurological signs and brain metabolic alterations (selective hyper-metabolism) suggested the involvement of the basal ganglia, mesial temporal lobes, and the brainstem. Conversely, the cerebral cortex demonstrated a rather diffuse hypo-metabolism, with relative sparing of sensorimotor areas, which appeared in association with 
clinically overt, rapidly progressive, cognitive decline. Importantly, the presence of this dual aspect combining areas of hyper-metabolism (possibly suggesting an inflammatory process) and other regions with decreased metabolic activity (where the subsequent development of brain atrophy points towards a neurodegenerative process) is distinctive and reminiscent of the alterations found in post-encephalitic parkinsonism [17]. In agreement with these findings, the clinical syndrome shown by the patients might resemble encephalitis lethargica, a neurological syndrome spread in the period 1916-1930, and firstly described by Von Economo in Vienna. The initial description of the disorder suggested the presence of three different variants in the acute phase, namely somnolent-ophthalmoplegic, hyperkinetic, and akinetic [18]. The patients here described manifested many of the characteristic symptoms of acute encephalitis lethargica, including cognitive decline, myoclonus, and ophthalmoplegia, even if with different clinical evolution (appearance of parkinsonism closer to the encephalitis symptoms). Von Economo also described the progression of this disease, in which he observed typical parkinsonian signs (hypertonia and bradykinesia), at times in association with less common features (kinesia paradoxa and oculogyric crisis) [18]. Similarly, we observed the appearance of parkinsonian features just after the encephalitic phase (Patient 1) and in concomitance with encephalitis (Patient 2). In addition, reduced, asymmetric, DaT-SPECT uptake in the putamina was also demonstrated in Patient 1. Even more interestingly, the seminal pathological observations made at that time confirmed the involvement of upper midbrain and substantia nigra as the most frequent anatomical targets, followed by the basal ganglia, pons, and medulla $[18,19]$. The affected areas demonstrated soft, edematous alterations and reddish discoloration, suggestive for inflammatory lesions, while modest degree of cortical atrophy was also present. The recent observation of similar neuropathological changes in the brainstem of 43 patients died of COVID-19 [20] suggests possible shared mechanisms.

So far, four other patients developing a parkinsonian syndrome soon (within few weeks) after COVID-19 were described [10,21-23], and the description of one of them is highly similar to our observations in Patient 2. In detail, Méndez-Guerrero and colleagues reported a COVID-19 patient who developed a hypokinetic-rigid syndrome in association with vertical gaze limitation. Importantly, in our patient, the detection of CSF-restricted oligoclonal bands points toward an immune-mediated (post-infectious) etiology. Conversely, the absence of clinically significant response following immunotherapy is possibly related to the subsequent development of neurodegeneration.

The link between autoimmunity and neurodegeneration was proposed also for other immune-mediated disorders that can present with atypical parkinsonism and sleep disorders including, for instance, IgLON5 autoimmune encephalitis. In particular, the pathological evidence of tau protein deposits suggested a subsequent irreversible brain damage which is likely triggered by the immune process [24, 25]. In this context, the altered amyloid $\beta 42$ and T-tau protein levels detected in the CSF of Patient 1, who showed dramatic decline of cognitive functions during the course of the disease, probably reflected extensive neuronal damage. This possibility is further supported by cortical thickness reduction found at the second MRI examination.

Interestingly, few other autoimmune encephalitides, including those targeting LGI1 and NMDAR antigens, showed similar FDG-PET/CT abnormalities [26, 27]. Maeder-Ingvar et al. reported a 25-year woman with antiNDMAR antibody encephalitis who developed limbs rigidity and akinetic mutism. FDG-PET showed diffuse cortical hypo-metabolism and bilateral basal ganglia hyper-metabolism which normalized after the improvement of movements disorders [26]. The pattern noted in our patients is also similar to the metabolic alterations reported by Delorme et al. [28] in four patients with COVID-19-related encephalopathy. However, none of the patients discussed by these Authors developed parkinsonism. Global cortical hypometabolism is characteristic of delirium, but Haggstrom et al. [29] reported only one case out of 13 patients (4/13 of which with dementia) with hyper-metabolism within the basal ganglia, and no case of hyper-metabolism within the brainstem and the cerebellum.

Remarkably, there was correspondence between cerebral areas with relatively increased cortical thickness and areas with hyper-metabolism, including the bilateral temporal pole and mesiotemporal region, suggesting their involvement during the inflammatory process. However, additional studies with serial neuroimaging examinations are needed to investigate the evolution between hyper- and hypo-metabolism and their structural correlates in MRI. In this regard, increased volume of gray matter has been reported in a subset of autoimmune limbic encephalitis during the early stages of the disease [30].

An immune-mediated etiology was hypothesized also for encephalitis lethargica: among 20 cases observed in 2004, $55 \%$ had a preceding infection (mostly pharyngitis), $69 \%$ showed the presence of oligoclonal bands in the CSF, and 95\% had autoantibodies reactive against human basal ganglia antigens [31].

An alternative possibility is that SARS-CoV-2 infection causes the hastening of pre-clinical neurodegenerative diseases, as it was suggested for one patient with newly diagnosed Parkinson's disease and previous history of premotor symptoms (constipation) [10] and one case of Creutzfeldt-Jakob disease occurring in tandem with symptomatic onset of COVID-19 [32]. We cannot exclude that the infection unmasked a pre-clinical parkinsonism especially 
in Patient 2, where the potential confounding role of previous treatment with neuroleptics should be acknowledged. However, our patients (i) lacked a previous history of prodromal parkinsonian symptoms, (ii) they did not show motor features prior to COVID-19, (iii) parkinsonism started subacutely in close temporal association with encephalitis, (iv) it did not improve following discontinuation of neuroleptics, and it did not benefit from levodopa.

The present study is obviously limited by the small sample size, but we believe that it provides novel clinical and PET data for the characterization of this still obscure disorder. Further limitations include the lack of IgLON5 antibody testing in our two cases, which can rarely present in a similar way. More observations are needed to determine whether the central nervous system, and in particular the brainstem and basal ganglia, are affected by SARS-CoV-2 or its immunemediated complications.

\section{Conclusion}

Findings from our study support the hypothesis that SARSCoV-2 infection can-in rare circumstances-trigger inflammatory and neurodegenerative processes leading to the development of parkinsonian symptoms and cognitive disturbances. In this context, PET imaging (especially if associated with objective automated analysis) may provide additional information on the brain areas affected and it can be particularly useful in patients with COVID-19-related encephalitis/encephalopathy and negative brain MRI. However, additional data on the metabolic patterns and their pathophysiologic basis are needed to assess the potential contribution of PET imaging in clinical practice.

\section{Declarations}

Conflict of interest The authors declare they have no known competing financial interests or personal relationships which could have influenced the work hereby reported.

Ethics statement Consent was obtained for publication by the relatives, and all identifiable information has been removed. All procedures were performed in accordance with the Declaration of Helsinki.

\section{References}

1. Farhadian S, Glick LR, Vogels CBF et al (2020) Acute encephalopathy with elevated CSF inflammatory markers as the initial presentation of COVID-19. BMC Neurol 20:248. https://doi.org/ 10.1186/s12883-020-01812-2

2. Pilotto A, Odolini S, Stefano Masciocchi S et al (2020) Steroidresponsive encephalitis in Covid-19 disease. Ann Neurol. https:// doi.org/10.1002/ana.25783
3. Khoo A, McLoughlin B, Cheema S et al (2020) Postinfectious brainstem encephalitis associated with SARS-CoV-2. J Neurol Neurosurg Psychiatry 91:1013-1014. https://doi.org/10.1136/ jnnp-2020-323816

4. Zambreanu L, Lightbody S, Bhandari M et al (2020) A case of limbic encephalitis associated with asymptomatic COVID-19 infection. J Neurol Neurosurg Psychiatry. https://doi.org/10. 1136/jnnp-2020-323839

5. Morassi M, Bagatto D, Cobelli M et al (2020) Stroke in patients with SARS-CoV-2 infection: case series. J Neurol. https://doi. org/10.1007/s00415-020-09885-2

6. Vogrig A, Bagatto D, Gigli GL et al (2020) Causality in COVID19-associated stroke: a uniform case definition for use in clinical research. J Neurol. https://doi.org/10.1007/s00415-020-10103-2

7. Vogrig A, Gigli GL, Bnà C, Morassi M (2021) Stroke in patients with COVID-19: clinical and neuroimaging characteristics. Neurosci Lett 743:135564. https://doi.org/10.1016/j.neulet. 2020.135564

8. Gigli GL, Vogrig A, Nilo A et al (2020) HLA and immunological features of SARS-CoV-2-induced Guillain-Barré syndrome. Neurol Sci 41:3391-3394. https://doi.org/10.1007/ s10072-020-04787-7

9. Toscano G, Palmerini F, Ravaglia S et al (2020) Guillain-Barré syndrome associated with SARS-CoV-2. N Engl J Med. https:// doi.org/10.1056/NEJMc2009191

10. Makhoul K, Jankovic J (2021) Parkinson's disease after COVID19. J Neurol Sci 422:117331. https://doi.org/10.1016/j.jns.2021. 117331

11. Merello M, Bhatia KP, Obeso JA (2021) SARS-CoV-2 and the risk of Parkinson's disease: facts and fantasy. Lancet Neurol 20:94-95. https://doi.org/10.1016/S1474-4422(20)30442-7

12. Venkatesan A, Tunkel AR, Bloch KC et al (2013) Case definitions, diagnostic algorithms, and priorities in encephalitis: consensus statement of the international encephalitis consortium. Clin Infect Dis 57:1114-1128. https://doi.org/10.1093/ $\mathrm{cid} / \mathrm{cit} 458$

13. Pagani M, Chiò A, Valentini MC et al (2014) Functional pattern of brain FDG-PET in amyotrophic lateral sclerosis. Neurology 83:1067-1074. https://doi.org/10.1212/WNL.0000000000000792

14. Fischl B, Dale AM (2000) Measuring the thickness of the human cerebral cortex from magnetic resonance images. Proc Natl Acad Sci USA 97:11050-11055. https://doi.org/10.1073/pnas.20003 3797

15. Reuter M, Schmansky NJ, Rosas HD, Fischl B (2012) Within-subject template estimation for unbiased longitudinal image analysis. Neuroimage 61:1402-1418. https://doi.org/10.1016/j.neuroimage. 2012.02.084

16. Calvini P, Rodriguez G, Inguglia F et al (2007) The basal ganglia matching tools package for striatal uptake semi-quantification: description and validation. Eur J Nucl Med Mol Imaging 34:1240-1253. https://doi.org/10.1007/s00259-006-0357-2

17. Lopez-Alberola R, Georgiou M, Sfakianakis GN et al (2009) Contemporary encephalitis lethargica: phenotype, laboratory findings and treatment outcomes. J Neurol 256:396-404. https://doi.org/ 10.1007/s00415-009-0074-4

18. Hoffman LA, Vilensky JA (2017) Encephalitis lethargica: 100 years after the epidemic. Brain 140:2246-2251. https://doi.org/ 10.1093/brain/awx177

19. Anderson LL, Vilensky JA, Duvoisin RC (2009) Review: neuropathology of acute phase encephalitis lethargica: a review of cases from the epidemic period. Neuropathol Appl Neurobiol 35:462-472. https://doi.org/10.1111/j.1365-2990.2009.01024.x

20. Matschke J, Lütgehetmann M, Hagel C et al (2020) Neuropathology of patients with COVID-19 in Germany: a post-mortem case series. Lancet Neurol 19:919-929. https://doi.org/10.1016/S14744422(20)30308-2 
21. Cohen ME, Eichel R, Steiner-Birmanns B et al (2020) A case of probable Parkinson's disease after SARS-CoV-2 infection. Lancet Neurol 19:804-805. https://doi.org/10.1016/S1474-4422(20) 30305-7

22. Faber I, Brandão PRP, Menegatti F et al (2020) Coronavirus disease 2019 and parkinsonism: a non-post-encephalitic case. Mov Disord 35:1721-1722. https://doi.org/10.1002/mds.28277

23. Méndez-Guerrero A, Laespada-García MI, Gómez-Grande A et al (2020) Acute hypokinetic-rigid syndrome following SARS-CoV-2 infection. Neurology 95:e2109-e2118. https://doi.org/10.1212/ WNL.0000000000010282

24. Gaig C, Graus F, Compta Y et al (2017) Clinical manifestations of the anti-IgLON5 disease. Neurology 88:1736-1743. https://doi. org/10.1212/WNL.0000000000003887

25. Sabater L, Gaig C, Gelpi E et al (2014) A novel non-rapid-eye movement and rapid-eye-movement parasomnia with sleep breathing disorder associated with antibodies to IgLON5: a case series, characterisation of the antigen, and post-mortem study. Lancet Neurol 13:575-586. https://doi.org/10.1016/S1474-4422(14) 70051-1

26. Maeder-Ingvar M, Prior JO, Irani SR et al (2011) FDG-PET hyperactivity in basal ganglia correlating with clinical course in anti-NDMA-R antibodies encephalitis. J Neurol Neurosurg Psychiatry 82:235-236. https://doi.org/10.1136/jnnp.2009.198697
27. Wegner F, Wilke F, Raab P et al (2014) Anti-leucine rich glioma inactivated 1 protein and anti-N-methyl-D-aspartate receptor encephalitis show distinct patterns of brain glucose metabolism in 18F-fluoro-2-deoxy-d-glucose positron emission tomography. BMC Neurol 14:136. https://doi.org/10.1186/1471-2377-14-136

28. Delorme C, Paccoud O, Kas A et al (2020) Covid-19-related encephalopathy: a case series with brain FDG-PET/CT findings. Eur J Neurol. https://doi.org/10.1111/ene.14478

29. Haggstrom LR, Nelson JA, Wegner EA, Caplan GA (2017) 2-18F-fluoro-2-deoxyglucose positron emission tomography in delirium. J Cereb Blood Flow Metab 37:3556-3567. https://doi. org/10.1177/0271678X17701764

30. Wagner J, Weber B, Elger CE (2015) Early and chronic gray matter volume changes in limbic encephalitis revealed by voxel-based morphometry. Epilepsia 56:754-761. https://doi.org/10.1111/epi. 12968

31. Dale RC, Church AJ, Surtees RAH et al (2004) Encephalitis lethargica syndrome: 20 new cases and evidence of basal ganglia autoimmunity. Brain 127:21-33. https://doi.org/10.1093/brain/ awh008

32. Young MJ, O'Hare M, Matiello M, Schmahmann JD (2020) Creutzfeldt-Jakob disease in a man with COVID-19: SARS-CoV2-accelerated neurodegeneration? Brain Behav Immun 89:601603. https://doi.org/10.1016/j.bbi.2020.07.007 